\section{Testículo evanescente en un paciente pediátrico: tratamiento laparoscópico}

Padilla-Piña J, García-Vásquez RA, López-Chente Casado J, Arriaga-Aguilar J, Zárate-Morales A, García De León-Gómez JM

\section{Resumen}

ANTECEDENTES: el síndrome de regresión testicular, o testículo evanescente, es una alteración originada por la atrofia y desaparición de un testículo sano durante el periodo fetal. Desde el punto de vista anatómico, se caracteriza por un cordón espermático con ausencia de tejido testicular macroscópicamente identificable. La ausencia de un testículo en un paciente con expresión 46XY suele ser unilateral y surge como consecuencia de una torsión o un infarto intrauterino o perinatal.

CASO CLÍNICO: paciente de 2 años 6 meses de edad, nacido del segundo embarazo, atendido para valoración por criptorquidia izquierda. A la exploración física se observaron los genitales de acuerdo con la edad y sexo, desarrollo gonadal, pene y prepucio normales; el testículo derecho en la bolsa escrotal (aproximadamente de $12 \mathrm{~mm}$ ) y el izquierdo no palpable. El ultrasonido inguinoescrotal mostró la bolsa escrotal izquierda vacía, sin identificación del testículo. La resonancia magnética evidenció sólo el testículo derecho en el escroto. La laparoscopia diagnóstica reportó, en el área inguinal derecha, el trayecto del conducto deferente sin alteraciones y vasos gonadales de calibre normal en el anillo inguinal; del lado izquierdo se encontró el conducto deferente con terminación ciega, con ausencia de vasos gonadales y anillo inguinal cerrado.

CONCLUSIÓN: la laparoscopia es el estudio de elección para establecer el diagnóstico de testículo evanescente, pues proporciona un mejor acceso a la cavidad abdominal y mayor visibilidad, además de recuperación posquirúrgica más rápida y aplicable a la edad pediátrica.

PALABRAS CLAVE: testículo evanescente, criptorquidia, laparoscopia pediátrica.

Rev Mex Urol. 2017 Sep-Oct;77(5):405-410.

\section{Vanishing testis in a pediatric patient: Laparoscopic management}

Padilla-Piña J, García-Vásquez RA, López-Chente Casado J, Arriaga-Aguilar J, Zárate-Morales A, García De León-Gómez JM

\section{Abstract}

BACKGROUND: Testicular regression syndrome, or vanishing testis, is an alteration caused by the atrophy and disappearance of a healthy
Servicio de Urología, Hospital General del Estado de Sonora (SSA), Universidad de Sonora (UniSon), Hermosillo.

Recibido: diciembre 2016

Aceptado: septiembre 2017

Correspondencia

José Padilla Piña

drpadillajose@gmail.com

Este artículo debe citarse como

Padilla-Piña J, García-Vásquez RA, López-Chente Casado J, Arriaga-Aguilar J, Zárate-Morales A, García De León-Gómez JM. Testículo evanescente en un paciente pediátrico: tratamiento laparoscópico. Rev Mex Urol. 2017 sep-oct;77(5):405-410. DOI: https://doi.org/10.24245/revmexurol.v77i5.1117 
testis during the fetal period. Anatomically, it is characterized by a spermatic cord with no macroscopically identifiable testicular tissue. The absence of a testis in a 46XY male is usually unilateral and is the result of torsion or of intrauterine or perinatal infarction.

CLINICAL CASE: A 30-month-old male child, product of a second pregnancy, was evaluated for left cryptorchidism. Physical examination revealed genitals in accordance with age and sex, good gonadal development, a normal penis and prepuce, a right testis of approximately $12 \mathrm{~mm}$ in the scrotal sac, and a nonpalpable left testis. Inguinoscrotal ultrasound image showed an empty left scrotal sac and identified no left testis. A magnetic resonance study showed only a right testis in the scrotum. Diagnostic laparoscopy revealed a normal vas deferens tract in the right inguinal area, as well as good caliber gonadal vessels in the inguinal ring. A blind-ending vas deferens was found on the left side, along with absent gonadal vessels, and a closed inguinal ring.

CONCLUSIONS: Laparoscopy is the diagnostic study of choice for nonpalpable testis, given that it provides better access to the abdominal cavity and greater visibility, along with faster postoperative recovery in both children and adults.

KEYWORDS: Vanishing testis; Cryptorchidism; Pediatric laparoscopy
Servicio de Urología, Hospital General del Estado de Sonora (SSA), Universidad de Sonora (UniSon), Hermosillo.

Correspondence José Padilla Piña drpadillajose@gmail.com

\section{ANTECEDENTES}

El síndrome de regresión testicular, o testículo evanescente, es una alteración originada por la atrofia y desaparición de un testículo durante el periodo fetal. ${ }^{1}$ La coexistencia de las estructuras del cordón espermático evidencian la presencia de los testículos en la vida intrauterina temprana. Cuando se identifica un cordón espermático con terminación ciega, esta alteración se denomina síndrome de testículo evanescente. Desde el punto de vista anatómico, se caracteriza por un cordón espermático con ausencia de tejido testicular macroscópicamente identificable. ${ }^{2}$ La ausencia de un testículo en un paciente con expresión 46XY suele ser unilateral y surge como consecuencia de alguna torsión o un infarto intrauterino o perinatal. ${ }^{3} \mathrm{El}$ síndrome de regresión testicular es una alteración poco frecuente, pues ocurre en menos de $5 \%$ de los casos de criptor- quidia. En pacientes con testículos no palpables, el testículo evanescente aparece en 35 a 60\% de los casos. Una revisión del Children's Hospital of Philadelphia relacionada con testículos no palpables mostró que $41 \%$ (187) de los niños tuvieron remanentes atróficos o ausencia testicular, por lo que se deduce es un fenómeno común no diagnosticado y, por tanto, raramente tratado.

\section{Etiología y fisiopatología}

Se han implicado factores hormonales y mecánicos en el descenso testicular; sin embargo, hasta el momento no existe un factor específico relacionado con la patogénesis de los testículos no palpables. El síndrome de regresión testicular se ha explicado como resultado de una trombosis, torsión o endocrinopatía, pero los estudios más recientes apoyan la idea de que se deriva a partir de un accidente vascular o torsión antenatal, en 
lugar de la endocrinopatía, debido a que los testículos no descendidos completamente son más propensos a la torsión durante el periodo fetal y perinatal. ${ }^{4}$ Esta hipótesis se comprueba por la identificación de macrófagos con alta concentración de hemosiderina, además de congestión venosa e infartos hemorrágicos secundarios a la torsión. Hay pacientes con testículo evanescente unilateral que no tienen concentraciones bajas de células germinales en el testículo descendido, sugerentes de endocrinopatía. ${ }^{9}$ La regresión testicular se ha relacionado con anormalidades genéticas, como microdeleciones del cromosoma Y, incluso se ha identificado persistencia de estructuras müllerianas después del nacimiento en niños con testículo evanescente. Además, la pérdida temprana de ambos testículos causa ambigüedad genital; sin embargo, aún no se establece la asociación entre factores genéticos con el descenso testicular, la anorquia y el testículo evanescente. En la mayoría de los casos el testículo evanescente suele ser esporádico y ocurre en pacientes con o sin antecedentes familiares de la alteración. Recientemente se ha reportado la asociación con otros defectos, principalmente retraso mental en hermanos cromosómicamente sanos o en varios miembros de la misma familia, lo que sugiere una base genética en algunos pacientes. ${ }^{5}$ Las manifestaciones clínicas del testículo evanescente pueden relacionarse con algunas de clasificaciones de secuencia embriológica: 1) embrionaria temprana y tardía, 2) fetal media y tardía, o 3) tempranamente neonatal. Esta alteración puede ser unilateral, incluso bilateral en casos excepcionales, con ausencia parcial o completa de tejido testicular y genitales externos normales. Los pacientes suelen tener fenotipo masculino o variar de masculino normal en caso de testículos no palpables unilaterales, masculinos fenotípicamente con micropene o fonotípicamente femeninos. ${ }^{6,7} \mathrm{Es}$ probable que el fenotipo dependa de la extensión y el momento del accidente intrauterino. El testículo evanescente de origen embrionario se considera parte clínica de la disgenesia gonadal
$46 \mathrm{XY}$, relacionado con micropene y testículo uni o bilateral. El grado de masculinización de los genitales también depende de la duración de la función testicular antes de su evanescencia. ${ }^{8} \mathrm{Se}$ han reportado algunos grados de ambigüedad sexual en casos familiares; recientemente se informó un caso de testículo evanescente con pérdida heterocigótica (V355M) en el gen SF1.

\section{CASO CLÍNICO}

Paciente masculino de 2 años 6 meses de edad, nacido de un segundo embarazo (madre de 34 años de edad, sana; padre de 38 años con artritis reumatoide), normoevolutivo, mediante vía vaginal, de 2900 g y 46 cm; con antecedente de infecciones de vías respiratorias superiores esporádicas. Acudió a valoración por criptorquidia izquierda; en la exploración física se encontraron: genitales de acuerdo con la edad y sexo, buen desarrollo gonadal, pene y prepucio normales, testículo derecho en la bolsa escrotal (de aproximadamente $0.5 \mathrm{~cm}$ ) y testículo izquierdo no palpable. El ultrasonido inguinoescrotal evidenció la bolsa izquierda vacía, sin identificación del testículo, y la bolsa escrotal derecha con visualización del testículo (Figura 1). La resonancia magnética mostró solamente el testículo derecho en el escroto (Figura 2). Se decidió realizar una laparoscopia diagnóstica y terapéutica por testículo izquierdo no palpable ni visible en los estudios de imagen. La cirugía se llevó a cabo con anestesia general; se preparó al paciente con sonda nasogástrica y vesical. El neumoperitoneo se efectuó con dióxido de carbono, mediante la técnica de Hasson, con la introducción intraperitoneal de un puerto de 5 mm con visión directa, a través de una incisión en la región umbilical. La presión intrabdominal de $\mathrm{CO}_{2}$ se llevó a $12 \mathrm{mmHg}$ como máximo, para la colocación de los trócares. A través del puerto umbilical se introdujo un lente de $5 \mathrm{~mm}$ conectado a una cámara, para visualización en el monitor de vídeo. El primer paso consistió en la exploración de la cavidad peritoneal, a 


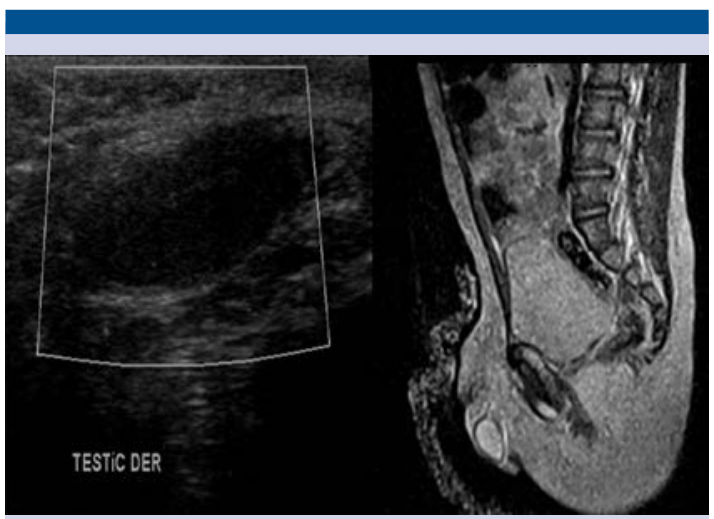

Figura 1. Ultrasonido inguinoescrotal que solo muestra el testículo derecho.

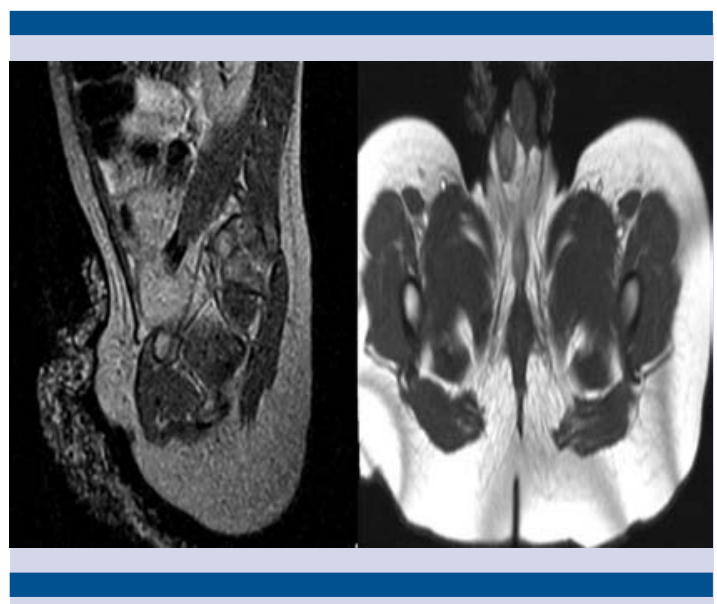

Figura 2. Resonancia magnética que evidencia la ausencia del testículo izquierdo.

continuación y con control videolaparoscópico se introdujeron dos puertos de $5 \mathrm{~mm}$ para instrumentación a cada lado, en la línea media clavicular. Se encontraron como referencias anatómicas los ligamentos vesicales laterales y los vasos iliacos; del lado derecho se identificó el trayecto del conducto deferente de forma normal y los vasos gonadales de buen calibre en el anillo inguinal (Figura 3A); del lado izquierdo se encontró el conducto deferente con terminación ciega, ausencia de vasos gonadales y anillo inguinal cerrado (Figura 3B). Se fulguró el conducto deferente izquierdo y se retiraron los trócares con visión directa. El tiempo quirúrgico fue de 28 minutos y el sangrado fue mínimo; no se registraron complicaciones durante el procedimiento quirúrgico, ni incidencias posoperatorias tempranas o tardías.

\section{DISCUSIÓN}

El testículo no palpable, también conocido como testículo evanescente o síndrome de regresión testicular, se caracteriza por ausencia parcial o total de tejido testicular; puede ser uni o bilateral, con o sin epidídimo o estructuras del cordón espermático y su expresión cromosómica es 46 XY. La exploración quirúrgica mediante laparoscopia representa el método de elección para establecer el diagnóstico y tratamiento, pues permite realizar la orquidopexia o resección de tejidos remanentes. Presentamos el caso de un paciente de edad pediátrica con testículo evanescente, en el que solo se encontró el conducto deferente con terminación ciega, sin tejido testicular. Existe

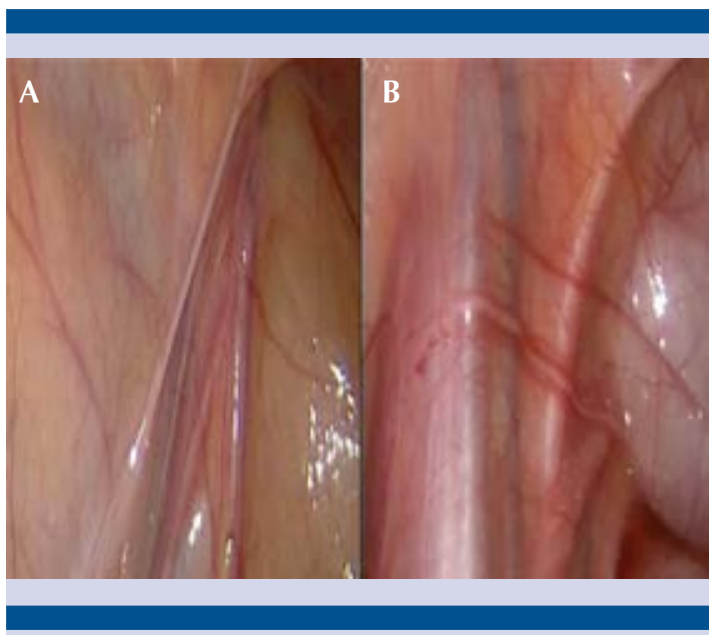

Figura 3. Estructuras anatómicas: conducto deferente con terminación ciega y ausencia de vasos gonadales (A); coexistencia del trayecto del conducto deferente y vasos gonadales de apariencia normal en el anillo inguinal (B). 
un alto riesgo de transformación maligna en los testículos intraabdominales, especialmente cuando se asocian con genitales externos anómalos y alteraciones cromosómicas.

El tumor testicular puede aparecer después de efectuar la orquidopexia; sin embargo, hasta el momento no se ha estimado el riesgo en los pacientes con testículo evanescente. Esta alteración comparte la misma fisiopatología y riesgos que la criptorquidia, incluso se discute su asociación con el tejido germinal residual y los remanentes testiculares, pues teóricamente indican un potencial de malignidad a largo plazo. Según distintas series, se han encontrado células germinales viables en $16 \%$ de los casos; con base en esto, algunos autores suelen remover los restos de forma rutinaria y otros no aceptan esta indicación, pues no han encontrado tejido testicular en los remanentes en la mayoría de los pacientes. ${ }^{9}$

Desde el punto de vista clínico, los testículos no palpables unilaterales pueden asociarse con testículos evanescentes, criptorquidia, testículos retráctiles o agenesia testicular. La laparoscopia es un estudio ampliamente utilizado para distinguir estas alteraciones y evitar la cirugía abierta. ${ }^{9}$

La laparoscopia pediátrica fue introducida en los primeros años de la década de 1970 por Steven Gans. La edad de los pacientes pediátricos no representa una limitante, pues en la actualidad se cuenta con ópticas, trócares y material quirúrgico desde $1.7 \mathrm{~mm}$ de diámetro, para su aplicación hasta en neonatos. La laparoscopia en pediatría es un procedimiento exploratorio muy conveniente y en algunos casos insustituible. ${ }^{6,10}$

Las principales ventajas de la cirugía laparoscópica son: recuperación posquirúrgica más rápida (debido al dolor postoperatorio mínimo), menor restricción en las actividades posoperatorias, cicatrización más estética y mejor visualización del campo operatorio. En pacientes con testículo no palpable, el conducto deferente y los vasos espermáticos pueden visualizarse saliendo del anillo inguinal interno y cuando sólo se encuentran remanentes, éstos pueden ser removidos. Diversos estudios reportan 90 a 95\% de efectividad con la laparoscopia para la localización de testículos no palpables en el examen físico. Algunos criterios propuestos para el diagnóstico certero de testículo evanescente incluyen: 1) testículo no palpable durante la exploración con anestesia, 2) visualización de vasos espermáticos con terminaciones ciegas sin visualización de vasos deferentes y vasos espermáticos, y 3) vasos deferentes con terminaciones ciegas y el anillo inguinal interno cerrado. Por lo tanto, solo se requiere la exploración inguinal cuando el anillo interno se visualiza abierto en la laparoscopia, no así cuando se encuentran vasos hipoplásicos y el anillo inguinal permanece cerrado.

El testículo evanescente representa un reto diagnóstico; su incidencia es variable, incluso desconocida. La cirugía de mínima invasión es una alternativa efectiva en pacientes pediátricos, principalmente en centros hospitalarios con experiencia, que dispongan de los recursos humanos y materiales necesarios. La laparoscopia es el método de elección para establecer el diagnóstico de testículo evanescente, pues proporciona un mejor acceso a la cavidad abdominal y mayor visibilidad, además de recuperación posquirúrgica más rápida, también aplicable en pacientes pediátricos.

\section{Financiamiento}

Los autores no recibieron ningún patrocinio para el desarrollo de esta investigación.

\section{Conflicto de interés.}

Los autores no tienen ningún conflicto de interés. 


\section{REFERENCIAS}

1. Sheikh A, Mirza B, Ahmad S, et al. Laparoscopic management of 128 undescended testes: Our experience. Afr J Paediatr Surg. 2012;9(2):106-108.

2. Park K, Choi H. An evolution of orchiopexy: historical aspect.Korean J Urol 2010;51(3):155-160.

3. López $P$, Angel L, Rodríguez J, et al. Abordaje laparoscópico en el testículo no palpable. Rev Chil Pediatr 2009;80(3):225-230.

4. Antic T, Hyjek E, Taxy J. The Vanishing Testis. A histomorphologic and clinical assessment. Am J Clin Pathol 2011;(136):872-880.

5. Shalaby M. Is there a role for open surgery in the management of the undescended testis?. Afr J Paediatr Surg. 2012;9(2):97-100.
6. Shalaby M, Shoma A, Elanany F, et al. Management of the looping vas deferens during laparoscopic orchiopexy. J Urol 2011;185(Suppl 6):2455-7.

7. Vipul G, Sunikumar Y, Elterman D, et al. Paediatric laparoscopic orchidopexy as a novel mentorship: Training model. Afr J Paediatr 2013;10(2):117-121.

8. EITayeb A. 2009. The unilateral impalpable testis: does the order of the procedure affect the outcome? Ann Pediatric Surg 2009;5:115-18.

9. Pirgon O, Dündar BN. Vanishing testes: A literature review. J Clin Res Pediatr Endocrinol 2012;4(3):116-120.

10. Hrivatakis $G$, Astfalk W, Schmidt A, et al. The timing of surgery for undescended testis a retrospective multicenter analysis. Dtsch Arztebl Int 2014;111(39):649-57.

\section{AVISO PARA LOS AUTORES}

Revista Mexicana de Urología tiene una nueva plataforma de gestión para envío de artículos: https://www.revisionporpares.com/index.php/RMUrol ahí podrá inscribirse a la base de datos administrada por el sistema Open Journal System (OJS) que ofrece las siguientes ventajas para los autores:

- Subir sus artículos directamente al sistema.

- Conocer, en cualquier momento, el estado de los artículos enviados, es decir, si ya fueron asignados a un revisor, aceptados con o sin cambios, o rechazados.

- Participar en el proceso editorial corrigiendo y modificando sus artículos hasta su aceptación final. 